determine the minimum inhibitory concentration (MIC) of these strains to AZM. All AZM-R isolates(MIC $\geq 2 \mu \mathrm{g} / \mathrm{ml}$ ) were screened for mutations in $23 \mathrm{~S}$ rRNA, $m$ trR and erm genes and genotyped using $N$. gonorrhoeae multi-antigen sequence typing (NG-MAST).

Results 50 (6.3\%) AZM-R isolates were collected, including 21 with azithromycin high-level resistant (AZM-HLR, MIC $\geq 256 \mu \mathrm{g} / \mathrm{ml}$ ) and 29 with azithromycin middle-level resistant (AZM-MLR, MIC between 2 to $16 \mu \mathrm{g} / \mathrm{ml}$ ). There were 18 strains had mutations (A2143G) and 12 strains had mutations (C2611T) in the four copies of the 23S rRNA gene in the AZ-HLR and AZ-MLR group respectively. Analysis of the promoter and coding region of $m t r R$ gene, significantly more AZM-HLR exhibited one pattern contained G45D and Y105H mutations compared with the AZM-MLR and AZ-S. Only one strains with positive PCR detection of ermB gene $(\mathrm{MIC}=2 \mu \mathrm{g} / \mathrm{ml})$. Among all the isolates, 81 sequence types (STs) were identified by NG-MAST, of which 8 STs were more often in the AZ-R group. Some STs (ST3356 and ST1866) that were observed had been noted in a previous reports of emerging AZM-R N. gonorrhoeae in Nanjing, Chongqing and Guangzhou. No resistant strains cluster was observed by NJ phylogenetic tree.

Conclusion AZM should not be recommended as a monotherapy for gonococcal in Shenzhen. The high-level and middlelevel resistance to AZM in $N$. gonorrhoeae were mainly mediated by specific mutations A2059G and C2611T in 23S rRNA respectively. Repeated emergence of ST1866 and ST3356 may help us to better monitor and analyse the epidemiological characteristics of AZM-R N. gonorrhoeae strains.

Support: Shenzhen Centre For Chronic Disease Control has been responsible for antimicrobial resistance surveillance program of N. gonorrhoeae in Shenzhen since 1992.

\section{LB3.245 GENOTYPING OF HIGH-RISK HUMAN PAPILLOMAVIRUS TYPES ASSOCIATED WITH PREDICTOR FACTORS TO CERVICAL CANCER IN WOMEN WITHOUT CYTOLOGICAL ABNORMALITIES: A CROSS SECTIONAL EPIDEMIOLOGICAL STUDY}

${ }^{1}$ Simões RSQ, ${ }^{2}$ Silva EP, ${ }^{1}$ Barth OM. 'Laboratory of Viral Morphology and Morphogenesis, Instituto Oswaldo Cruz, Fundação Oswaldo Cruz, Rio de Janeiro, RJ, Brazil, ${ }^{2}$ Vale do Itajaí University, Itajaí, Santa Catarina, Brazil

\subsection{6/sextrans-2017-053264.480}

Introduction The mucosal Human Papillomavirus (HPV) types have been described in the Alphapapillomavirus genus and infect the anogenital epithelium. There are viral groups based on their oncogenic activity as high-risk types, low-risk types and types of undetermined-risk. The aim of the study was to evaluate HPV frequency and co-factors related to cervical cancer in sexually active women from Rio de Janeiro city. This cross-sectional design study was performed at public health institutions from 2014 to 2016.

Methods Clinical samples were collected with a cervical cytobrush and stored at $-20^{\circ} \mathrm{C}$. After nucleic acid amplification, specific primers of high-risk types (HPV-16,-18,-31/-45) were used. Statistical analysis was applied in 18 socio-demographic variables.

Results This study demonstrated that of the 100 participants, $20 \%$ were DNA HPV positive. Of these, $8 \%$ were amplified by HPV-18/-45; and $12 \%$ were undetermined types. HPV-16 and -31 types were not detected. Comparing the genotypes,
$37.5 \%$ were predominately over 45 years old, $62.5 \%$ of respondents were HPV-18 positive, married and/or of co-habitation matrimonial status and62.5\% don't smoke. All of them don't use drugs and alcohol, four patients (50\%) have monthly income between one to four minimum wages, $37.5 \%$ started sexual activity before the age of 17 years and $50 \%$ had less than five sexual partners. Twenty five percent of the patients have had multiple infections and were unmarried. The 3\% who have had the both types of HPV infection had more than five sexual partners. A highly significant factor associated with HPV infection was who did not use the condom and had an income between one to four minimum wages, both with $87.5 \%$.

Conclusion This epidemiological study showed a strongly association among the presence of DNA HPV types with risk factors in unimmunized women. A large challenge to public health is cancer prevention in populations from developing countries with high risk exposure associated with the history of other sexually transmitted diseases (STDs) in different geographic regions.

Support: Coordenação de Aperfeiçoamento de Pessoal de Nivel Superior (CAPES)/Programa Brasil Sem Miséria; Conselho Nacional de Desenvolvimento Científico e Tecnológico (CNPq)

\section{LB3.246 PREVALENCE AND RISK FACTORS OF HUMAN PAPILLOMAVIRUS INFECTION IN UNIMMUNIZED WOMEN IN BRAZIL}

${ }^{1}$ Simões, RSQ, ${ }^{2}$ Silva EP, 'Barth OM. ' ${ }^{1}$ Laboratory of Viral Morphology and Morphogenesis, Instituto Oswaldo Cruz, Fundação Oswaldo Cruz, Rio de Janeiro, RJ, Brazil; ${ }^{2}$ Vale do Itajaí University, Itajaí, Santa Catarina, Brazil

10.1136/sextrans-2017-053264.481

Introduction The Brazilian Immunisation Program recommended the vaccine to be administered at 9 to 11 years of age before most adolescents became sexually active.The aim of this study is to describe the human papillomavirus(HPV) infection prevalence among unimmunized women and to identify the risk factors as demographic, behavioural and biological variables.

Methods An epidemiological study was performed in women randomly selected during 2014 to 2016. About 100 cervical cell scrapings were collected with a cytobrush. DNA extraction and HPV detection were performed by PCR, Nested PCR and specific primers. DNA quality was amplified by $\beta$-globin PCR primers. Restriction fragment length polymorphism (RFLP) patterns of L1 PCR products were used for genotyping.

Results In the population studied, HPV prevalence was of $20 \%$. HPV 18 and 45 were the most frequently detected HPV types. A group of100 women was divided into six aged groups ( $\leq 25$ y.o; 26 to 30 y.o; 31 to 35 y.o; 36 to 40 y.o; 41 to 45 y.o; $\geq 45$ y.o).Distribution among racial/ethnic groups was representative when $47 \%$ of women were white and $53 \%$ were black and on the race/ethnicities. Most women were currently not married (56\%) and married or cohabitating (44\%) $(\mathrm{p}<0,05)$.Psychosocial and psychosexual issues demonstrated that six percent of the women exhibit history of sexually transmitted diseases, except HIV, with $85 \%$ of women having sex with until to five partners $(p<0,03)$. About the employment status of all the participating women, $90 \%$ reported having had at least one until four basic salaries. Only 5\% women have had higher education. The most women (87\%) have had secondary and fundamental education. 